\title{
THE BINOMIAL QUARTIC AS A NORMAL FORM*
}

\section{BY RAYMOND GARVER}

From the standpoint of the Tschirnhausen transformation, the quartic equation may be said to occupy a distinct position, between the quadratic and cubic on one hand, and the higher degree equations on the other. For the lower degree equations can be reduced easily to binomial form by simple linear or quadratic transformations. Such reduction is not, in general, possible; but, for equations of degree not less than five, the second, third, and fourth terms can be removed by a fourth degree transformation without solving any equations of degree greater than the third. $\dagger$ (For the quintic such a transformation leads to the Bring-Jerrard normal form $x^{5}+a_{4} x+a_{5}=0$.) Now these two problems, reduction to binomial form, and removal of three intermediate terms, become equivalent for, and only for, the quartic equation. Hence the question of the reducibility of the quartic to the form $x^{4}+a_{4}=0$ is one of some interest.

It is worth while mentioning that none of the known methods for removing three terms apply to the quartic. They require, as mentioned above, fourth degree transformations, and fail when the given equation is of only that degree. $\ddagger$

An examination of the literature shows that the question of the reducibility of the general quartic to binomial form by means of a Tschirnhausen transformation has been considered

* Presented to the Society, October 29, 1927.

$\dagger$ See Dickson, Modern Algebraic Theories, 1926, Chap. XII; or almost any higher algebra.

$\ddagger$ Salmon, Modern Higher Algebra, 4th ed., 1885, p. 250, and Matthiessen, Grundzüge der Antiken und Modernen Algebra, 1878, p. 125, state incorrectly that three terms can be removed from a quintic (or higher degree equation) by a third degree transformation, without solving any equations of degree greater than three. 
by two men, and that they have stated different results. Lagrange, in his well known Réflexions sur la Résolution Algébrique des Equations, ${ }^{*}$ gives an à priori proof that the reduction is possible. If we consider

$$
x^{4}+a_{1} x^{3}+a_{2} x^{2}+a_{3} x+a_{4}=0,
$$

and the transformation

$$
y=x^{3}+k_{2} x^{2}+k_{3} x+k_{4},
$$

the conditions that the new equation in $y$ lack all intermediate terms are, as well known,

$$
\sum y=0, \quad \sum y^{2}=0, \quad \sum y^{3}=0,
$$

where the summations extend over the four roots of (1). The equations of condition are of degree 1,2 , and 3 respectively in the parameters $k_{2}, k_{3}, k_{4}$, and if we eliminate any two of them, say $k_{3}$ and $k_{4}$, we shall arrive at a sixth degree equation. What Lagrange does, by an elegant though somewhat lengthy process, is to show that this sixth degree equation (when the original equation is a quartic) factors into three quadratics whose coefficients are themselves roots of cubic equations.

Over a hundred years later Sylvester, apparently unaware of this part of Lagrange's work, stated the opposite conclusion. On page 548 of his paper On the so-called Tschirnhausen transformation $\dagger$, he says "Five is the minimum degree of equation from which three terms can be removed without solving an equation above the third degree," and a similar statement had been made earlier by Hamilton. $\ddagger$ However, the proof of Sylvester is incomplete, or rather he is considering a slightly different problem, that of deter-

\footnotetext{
* Published in 1770-71. See his Oeuvres, vol. III, Paris, 1869, especially pp. 284-295.

† Journal für Mathematik, vol. 100 (1887), pp.465-86. Collected Papers, vol. 4, Cambridge, 1912, pp. 531-49.

¥ Sixth Report of the British Association for the Advancement of Science, 1837, pp. 295-348.
} 
mining in general how many parameters must be present to insure a solution of a system of equations without introducing any elevation of degree. He does not consider the possibility, in special cases, of a factorization such as the one established by Lagrange for the quartic.

I wish to indicate a reduction of the quartic to binomial form entirely different in form from that of Lagrange, and which, making use of some rather lengthy calculations which have been already worked out, seems much simpler. It makes use of a special type of Tschirnhausen transformation introduced by Hermite* and used later by Cayley, $\dagger$ in which the coefficients of the transformed equation are invariants. The use of the special transformation is by no means essential, but is very convenient because the transformed equation has been calculated, and been put in convenient form. Briefly, if we take our quartic in the form

$$
a x^{4}+4 b x^{3}+6 c x^{2}+4 d x+e=0,
$$

and apply the transformation

$$
\begin{aligned}
y=(a x+b) B+( & \left.a x^{2}+4 b x+3 c\right) C \\
+ & \left(a x^{3}+4 b x^{2}+6 c x+3 d\right) D,
\end{aligned}
$$

then the second coefficient of the transformed equation will be zero, and the other coefficients will be invariants of the two forms $(a, b, c, d, e)(X, Y)^{4},(B, C, D)(Y,-X)^{2}$. The transformed equation has been computed by Cayley and Salmon;§ we do not need its explicit form. However we do need a result stated by Salmon;\| namely, that the invariant

* Comptes Rendus, vol. 46 (1858), p. 961; Oeuvres, vol. 2, Paris, 1908, pp. $30-37$.

$\dagger$ On Tschirnhausen's transformation, Collected Papers, vol. 4, Cambridge, 1891, pp. 375-94. See also Journal für Mathematik, vol. 58 (1861), pp. 263-69.

$\ddagger$ Cayley, loc. cit.

$\S$ Their notations are different; I have followed Cayley above, but have left (5) in Salmon's notation.

|| Loc. cit., p. 254. 
$a c e+2 b c d-a d^{2}-e b^{2}-c^{3}$, which he calls $T$, becomes, for the new equation,

$$
T \phi^{3}+S^{2} \Delta \phi^{2}+9 \Delta^{2} S T \phi+\Delta^{3}\left(54 T^{2}-S^{3}\right),
$$

where

$$
\begin{gathered}
S=a e-4 b d+3 c^{2}, \quad \Delta=(2 / 3)(B D-C)^{2}, \\
\phi=a B^{2}+4 b B C+c\left(2 B D+4 C^{2}\right)+4 d C D+e D^{2} .
\end{gathered}
$$

If we denote (5) by $T_{y}$, the condition $T_{y}=0$ is a cubic in $\phi / \Delta$ with known coefficients, which can then be solved. We then have to satisfy an equation of form $\phi / \Delta=r$, which, by (6), can be done by suitable choice of $B, C, D$. We then have the general quartic reduced to one in which the invariant $T$ is zero.

Now it is known* that any quartic whose invariant $T$ (known in algebraic geometry as the catalecticant) vanishes, can be reduced, by a linear fractional transformation (which is equivalent to a Tschirnhausen transformation), to binomial form. I have carried out the calculation, without any reference to the geometrical side of the problem, and while seemingly rather difficult it can be put in convenient form. Finally, since the product of two Tschirnhausen transformations is itself a Tschirnhausen transformation, we have the desired reduction.

\section{The UnIVERsity OF ROCHester}

* Winger, Introduction to Projective Geometry, 1923, p. 209. 